\title{
Antimony Resistance in Leishmania Parasites may be Related to an Increased Virulence
}

\author{
Laísa Vilar Cordeiro $^{1 *}$, Francisco Patricio de Andrade Júnior ${ }^{2}$ and Edeltrudes de Oliveira \\ Lima $^{3}$
}

${ }^{1}$ PhD student in Natural and Synthetic Bioactive Products at Federal University of Paraíba, Brazil

${ }^{2}$ Master in Natural and Synthetic Bioactive Products at Federal University of Paraíba, Brazil

${ }^{3}$ PhD Professor at Federal University of Paraíba, Brazil

*Corresponding author: Laísa Vilar Cordeiro, Department of Pharmaceutical Sciences, Health Sciences Center, Federal University

of Paraíba, Campus I, Castelo Branco, João Pessoa, Paraíba, Brazil

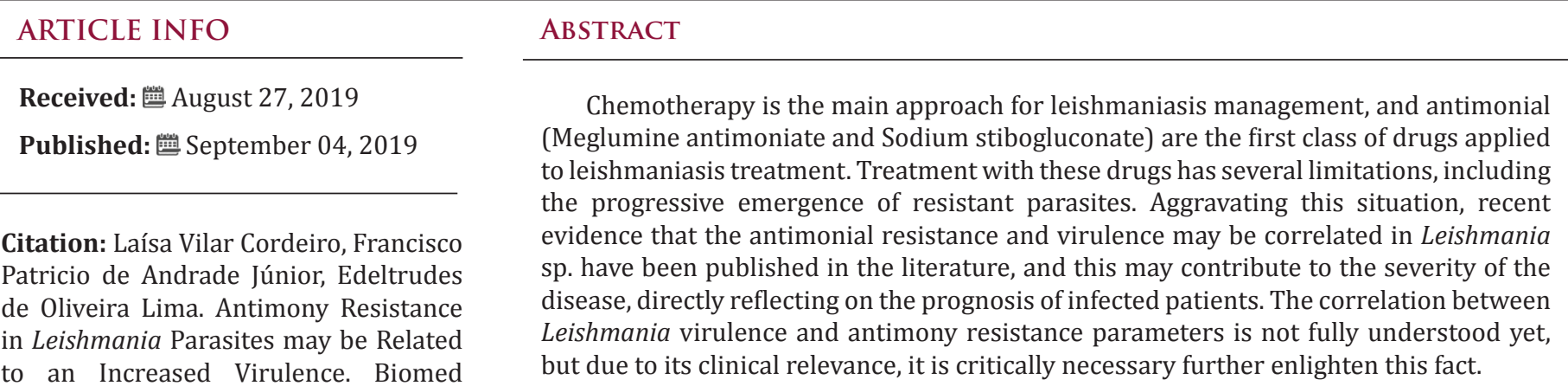

to an Increased Virulence. Biomed J Sci \& Tech Res 21(1)-2019. BJSTR. MS.ID.003555.

\section{Introduction}

The leishmaniasis are complex heterogenic vector-borne diseases caused by Leishmania spp. (Protozoa, Kinetoplastida, Trypanosomatidae) and spread by female sand flies [1]. Chemotherapy is the main approach for leishmaniasis management. However, the combined problems of parenteral administration and anti-leishmaniasis substantial side effects are factors that hinder treatment. In addition, the selection of resistant parasites carrying genetic mutations that lessen the parasite's response to drugs may emerge upon mass drug administration [2]. There are only a few drugs available for leishmaniasis treatment and most of them have been in use for quite some time. Pentavalent antimonial, amphotericin B, pentamidine, miltefosine and paromomycin constitute the resources available for leishmaniasis chemotherapy [3]. Antimonial (Meglumine antimoniate and Sodium stibogluconate) are the first class of drugs applied to leishmaniasis treatment. These toxic compounds have a narrow therapeutic window but are still in use in various regions of the world such as Latin America and East Africa [2,3].
Another complicating factor includes the fact that Leishmania parasites present the possibility of suffering considerable genomic changes, such as chromosomal aneuploidies, and changes in gene expression pattern, which appear to be involved in the adaptation of these organisms to the presence of the drug. Over the last 10 to 20 years, there has been an increased in clinical resistance to antimonial, which has even led to the abolition of use in some regions, for example, in North Bihar in India [2]. Some studies on prokaryotes and eukaryotes show that drug resistance is usually related to a reduction in the fitness of microorganisms, since there is a decrease in growth, virulence and transmissibility between hosts $[4,5]$. However, it has recently been observed that some species of Leishmania, especially L. donovani, resistant to antimonial appear to have developed a series of molecular adaptations that lead to an increased parasite fitness even in the absence of the drug. This ability has been related to the competence to generate metacyclic forms, which have greater power of host infection. The plastic nature of the genome of these parasites makes them highly 
adaptable, being able to modulate gene expression through gene amplification or deletion and alter chromosomal ploidy in response to drug-stress [6-9].

\section{Antimony Resistance and Increased Virulence}

Vanaerschot and collaborators [6] analyzed antimonial susceptible and resistant clinical isolates of $L$. donovani. The authors observed that resistant parasites showed lower sensitivity to oxidative and Nitrosative stress in vitro. The in vitro macrophage infection rate was significantly higher in drug-resistant strains and they were also more infective in vivo, reaching a higher parasitic load in both spleen and liver of infected Balb/c mice. The authors suggest that higher stress resistance and a greater number of metacyclic forms may contribute to resistant strains had superior survival skills as promastigotes and as amastigotes compared to the sensitive's strains. Ouakad and colleagues [7] demonstrated that antimony-resistant L. donovani strains had twice as many metacyclic forms as those found in antimony-sensitive cultures. Resistant cultures also had higher infection capacity in murine macrophages and were more resistant to complement-mediated lysis. Another study also analyzed antimony-resistant L. donovani strains found higher in vivo infection power [8]. Another research, by Moura and colleagues [9], used L. infantum strains isolated from refractory patients to antimony treatment (relapse cases) and responsive patients (control group). Macrophage infection was higher with $L$. infantum isolates from relapse cases and correlated with enhanced interleukin 1- $\beta$ production, furthermore, these parasites stimulated inflammatory cytokines and were resistant to macrophage killing mechanisms, factors that may contribute to disease severity.

\section{Conclusion}

Findings between the possible correlation of increased virulence in antimonial-resistant Leishmania parasites raise a

\section{ISSN: 2574-1241}

DOI: 10.26717/BJSTR.2019.21.003555

Laísa Vilar Cordeiro. Biomed J Sci \& Tech Res

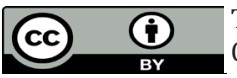

This work is licensed under Creative Commons Attribution 4.0 License

Submission Link: https://biomedres.us/submit-manuscript.php fundamental question about potential risks of selecting more virulent pathogens through massive chemotherapy interventions. Further work is needed to further clarify this fact and to lead to better guidelines for the treatment of these disorders.

\section{References}

1. Bates PA (2018) Revising Leishmania's life cycle. Nature microbiology 3(5): 529-530.

2. Ponte Sucre A, Gamarro F, Dujardin J, Barrett MP, López Vélez R, et al. (2017) Drug resistance and treatment failure in leishmaniasis: A 21 $1^{\text {st }}$ century challenge. Plos Negl Trop Dis 11(12): e0006052.

3. Uliana SRB, Trinconi CT, Coelho AC (2017) Chemotherapy of leishmaniasis: present challenges. Parasitology 145(4): 464-480.

4. Babiker HA, Hastings IM, Swedberg G (2009) Impaired fitness of drug-resistant Malaria parasites: evidence and implication on drugdeployment policies. Expert Rev Anti Infe 7(5): 581-593.

5. Abdelraouf K, Kabbara S, Ledesma KR, Poole K, Tam VH (2011) Effect of multidrug resistance-conferring mutations on the fitness and virulence of Pseudomonas aeruginosa. J Antimicrob Chemother 66(6): 1311-1317.

6. Vanaerschot M, Maes I, Ouakad M, Adaui V, Maes L, et al. (2010) Linking in vitro and in vivo survival of clinical Leishmania donovani strains. Plos one 5(8): e12211.

7. Ouakad M, Vanaerschot M, Rijal S, Sundar S, Speybroeck N, et al. (2011) Increased metacyclogenesis of antimony-resistant Leishmania donovani clinical lines. Parasitology 138(11): 1392-1399.

8. Vanaerschot M, Doncker S, Rijal S, Maes L, Dujardin JC, et al. (2011) Antimonial resistance in Leishmania donovani is associated with increased in vivo parasite burden. Plos One 6(8): e23120.

9. Moura TR, Santos MLB, Braz JM, Santos LF, Aragão MT, et al. (2016) Cross-resistance of Leishmania infantum isolates to nitric oxide from patients refractory to antimony treatment and greater tolerance to antileishmanial responses by macrophages. Parasitol Res 115(2): 713721.

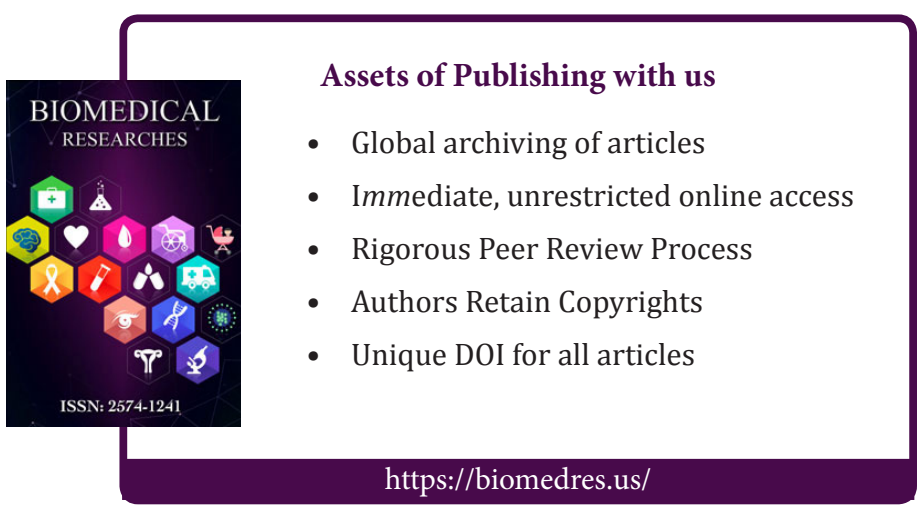

